\title{
STRUCTURAL AND OPTICAL INVESTIGATION OF CdSe QUANTUM DOTS
}

\author{
Neupane Dipesh \\ Department of Physics, Banaras Hindu University \\ Corresponding address: dipeshneupane07@gmail.com \\ Received 03 June, 2010; Revised 21 August, 2012
}

\begin{abstract}
CdSe semiconducting Quantum dots were prepared by a chemical method at a room temperature. X-ray powder diffraction and transmission electron microscope measurements conformed a hexagonal cubic crystalline phase of Cdse semiconducting Quantum dots of about $15 \mathrm{~nm}$ average size with nearly spherical shape. The absorption and photoluminescence spectra of the CdSe quantum dots were strongly shown blue shifted due to size quantization. The present study describes a simultaneous and highly reproducible large scale synthesis of highly luminescent CdSe Quantum dots.
\end{abstract}

\section{INTRODUCTION}

Synthesis of semiconducting Quantum dots are of great interest recently for both fundamental research and technical applications. Due to their size dependent physical and chemical properties such as work function, geometrical shape and structure, energy gap, bond length, etc ${ }^{(1-3)}$, which are very different from their bulk values. They are very much applicable in light emitting diodes (LED) ${ }^{(4)}$, biological labeling, solar cells ${ }^{(5)}$, etc. Among semiconducting materials, II-IV group semiconducting Quantum dots, especially CdSe Quantum dots have been deeply and systematically researched due to the well established synthetic technique. The method for synthesis of CdSe quantum dots dated from $\sim 1986^{(6-7)}$. The standard band gap value for CdSe Bulk semiconductor is $1.74 \mathrm{eV}$. When the crystal structure is less than Bohr radius, the quantum confinement effect becomes visible; its optical spectrum is shifted from red to blue as the crystal radius decreases. Different routes have been employed to synthesize CdSe quantum dots. These methods include electrochemical ${ }^{(8)}$, laser ablation ${ }^{(9)}$, surfactant- assisted hydrothermal ${ }^{(10)}$ etc. here, I present chemical method at room temperature which is novel. The Qd's were characterized by X-ray Diffraction, Transmission Electron Microscopy, UV/PL.

\section{EXPERIMENTAL SECTION}

Materials; All reagents used in this work including highly pure $\mathrm{CdCl}_{2}$ powder (99.9\%), highly pure elemental Selenium (99.9\%) purchased from Alfa, India were used without further purification. Ethylene glycol and Hydrazine hydrate purchased from Merch, Germany were used directly without any purification.

Synthesis of CdSe Quantum Dots; $\mathrm{CdCl}_{2}$ powder $(5.0 \mathrm{~g})$ and Elemental Selenium(2.0g) were taken with $70 \mathrm{ml}$ of deionized water to form a transparent solution 'A', at room temperature. Separately, ethylene glycol of $20 \mathrm{ml}$ and hydrazine hydrate of $20 \mathrm{ml}$ were added, to form solution 'B'. Then, solution 'A' and solution 'B' were mixed and refluxed under vigorous stirring at $70^{\circ} \mathrm{C}$ for 5 hours. Finally, the Brown precipitates were collected and washed with anhydrous ethanol for several times, then dried in vacuum at $50^{\circ} \mathrm{C}$ for 2 hours. 
Characterization; The sample was characterized by an advance $\mathrm{x}$-ray diffractometer (XRD) with $\mathrm{CuK} \alpha$ radiation $\left(\lambda=1.54 \AA\right.$ ). the $2 \Theta$ range used was from $10^{\circ}$ to $80^{\circ}$ in the step of $0.05^{\circ}$ with a count tome of $1 \mathrm{sec}$. the size of CdSe nano structure was measured by HRTEM operated at 150 KV. Optical diffuse reflectance of the sample was recorded on UV Spectrophotometer. Photoluminescence experiments were conducted in air on fluorescence spectrophotometer.

\section{RESULTS AND DISCUSSION}

\section{XRD studies:}

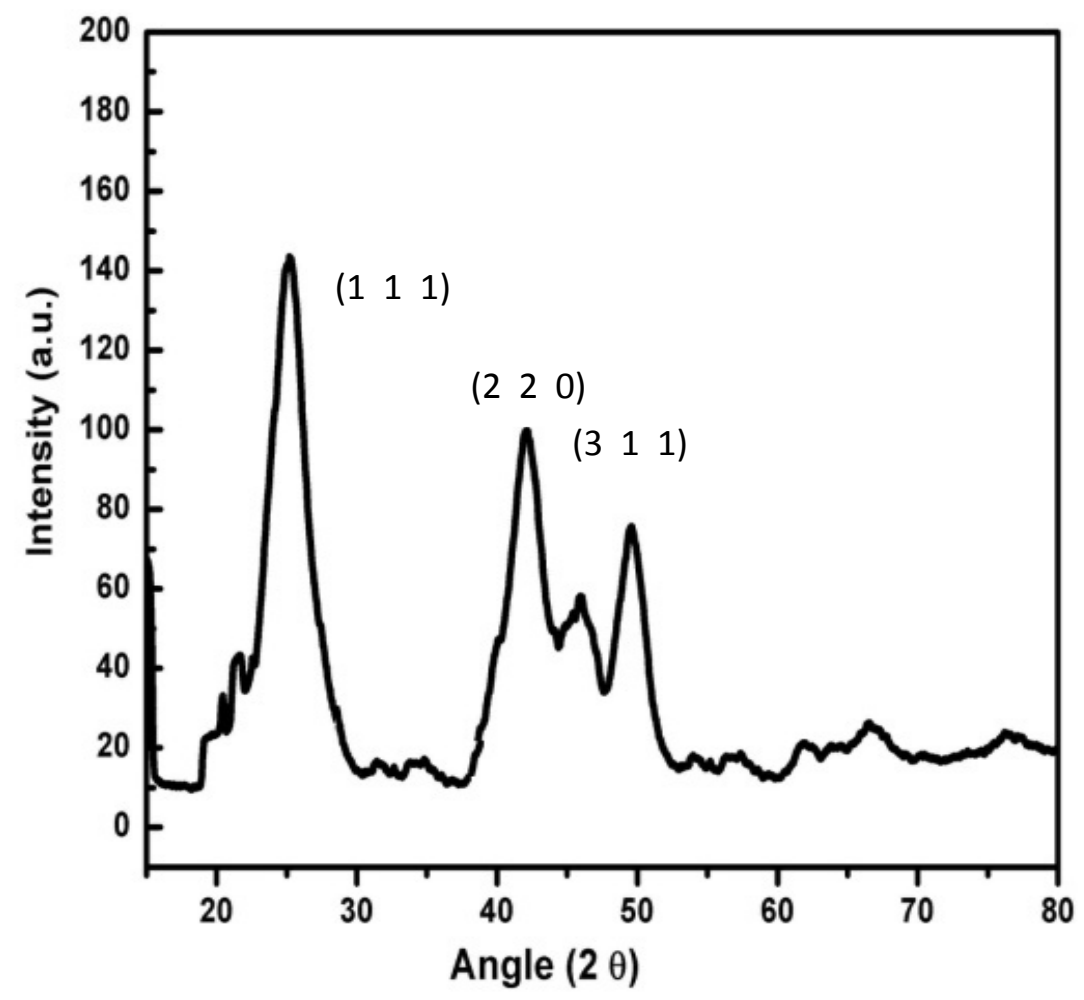

Figure 1. XRD pattern of CdSe nano structure

XRD diffraction pattern of the prepared CdSe sample is shown in fig 1 . The peaks of CdSe have been obtained due to diffraction corresponding to (1 111$),\left(\begin{array}{lll}2 & 2 & 0\end{array}\right),\left(\begin{array}{lll}3 & 1 & 1\end{array}\right)$ reflections, which is in a very good agreement with cubic CdSe structure, (Joint Committee on powder diffraction standers) JCPDS card no. 19-0191, CAS No. 1306-24-7, $(a=6.077 \AA=b=c)$. the information on the particle size (A) of CdSe Qd's has been obtained from the following Scherrer relations ${ }^{(11)}$;

$$
\mathrm{A}=0.94 \lambda / \beta \cos \theta
$$


Where $\mathrm{A}$ is coherence length, $\beta$ is the full-widths-at-half-maximum (FWHM) of the diffraction peak, $\lambda(1.5418 \AA)$ is the wavelength of the $\mathrm{X}$-ray radiation and $\theta$ is the angle of diffraction. The average size of CdSe determined by Scherrer relations was approximately $20 \mathrm{~nm}$.

UV - Visible and Photoluminescence Spectroscopy;

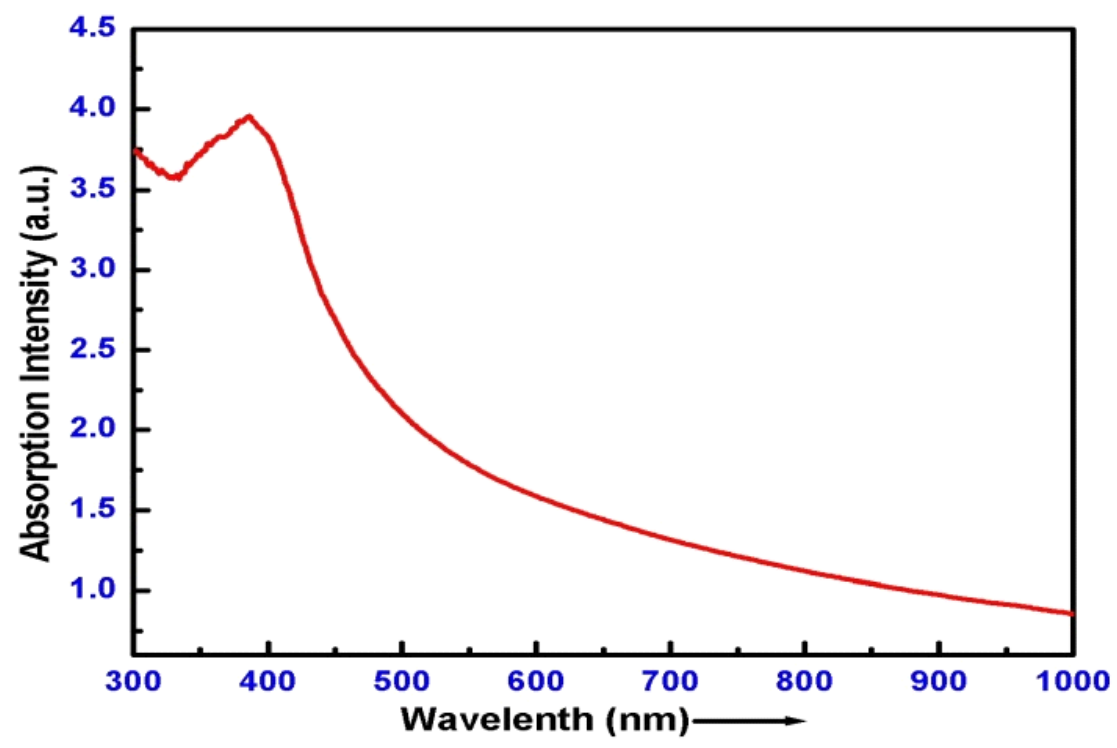

Figure 2. UV-Vis. spectrum of CdSe Sample at Room temperature

The optical properties of CdSe Qd's were characterized by UV - Vis absorption and the results are shown in fig. 2. There is an obvious shoulder peak in the UV - Vis absorption spectrum, and the corresponding onset at $380 \mathrm{~nm}$ can be assigned to the band gap and second excited state within each crystallite, respectively. The absorption spectra of CdSe nano particles were studied without taking into account of the reflection and transmission losses. Using it, the calculated band gap energy was $2.55 \mathrm{eV}$ (band gap increases as the particle size decreases ${ }^{(12)}$ ) which showed the blue shift of $0.8 \mathrm{eV}$ from standard bulk band gap energy $\left(\mathrm{E}_{\mathrm{g}}=1.7 \mathrm{eV}\right)$. As the size decreased, their optical properties varied and the wavelength range of the CdSe Qdots exhibited a blue shift ${ }^{(13)}$. The reaction conditions such as heating time, temperature, kinds and amounts of reagents have effect on the morphology and size of the products in the processes.

Similarly global Photoluminescence Spectra of CdSe quantum dots were recorded with the help of a computer controlled rationing luminescence spectrometer (LS55-Perkin Elmer Instruments, UK). Fig 3 shows the Photoluminescence (PL) spectra of CdSe nano structure. the near edge emission was observed near $457 \mathrm{~nm}$. The broad emission peak visible is assigned to blue emission $^{(13)}$ (larger the size of dots, the redder its fluorescence spectrum, conversely, smaller dots emit bluer light). 


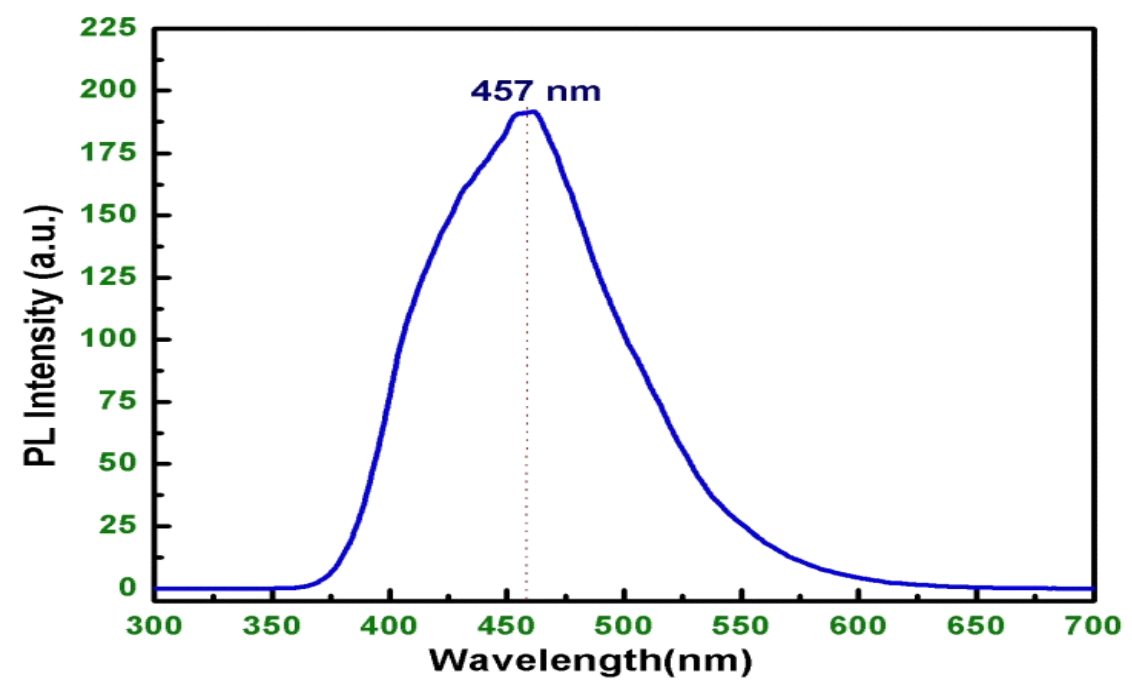

Figure 3. PL spectrum of CdSe Sample at Room temperature

\section{Electron microscopic characterization;}

From the resulting TEM image, the uniform particles image could be clearly observed in fig 4. On the basis of TEM image of CdSe sample, we obtain particle shape is nearly spherical and an average size of particles ranging from 15 to $20 \mathrm{~nm}$.

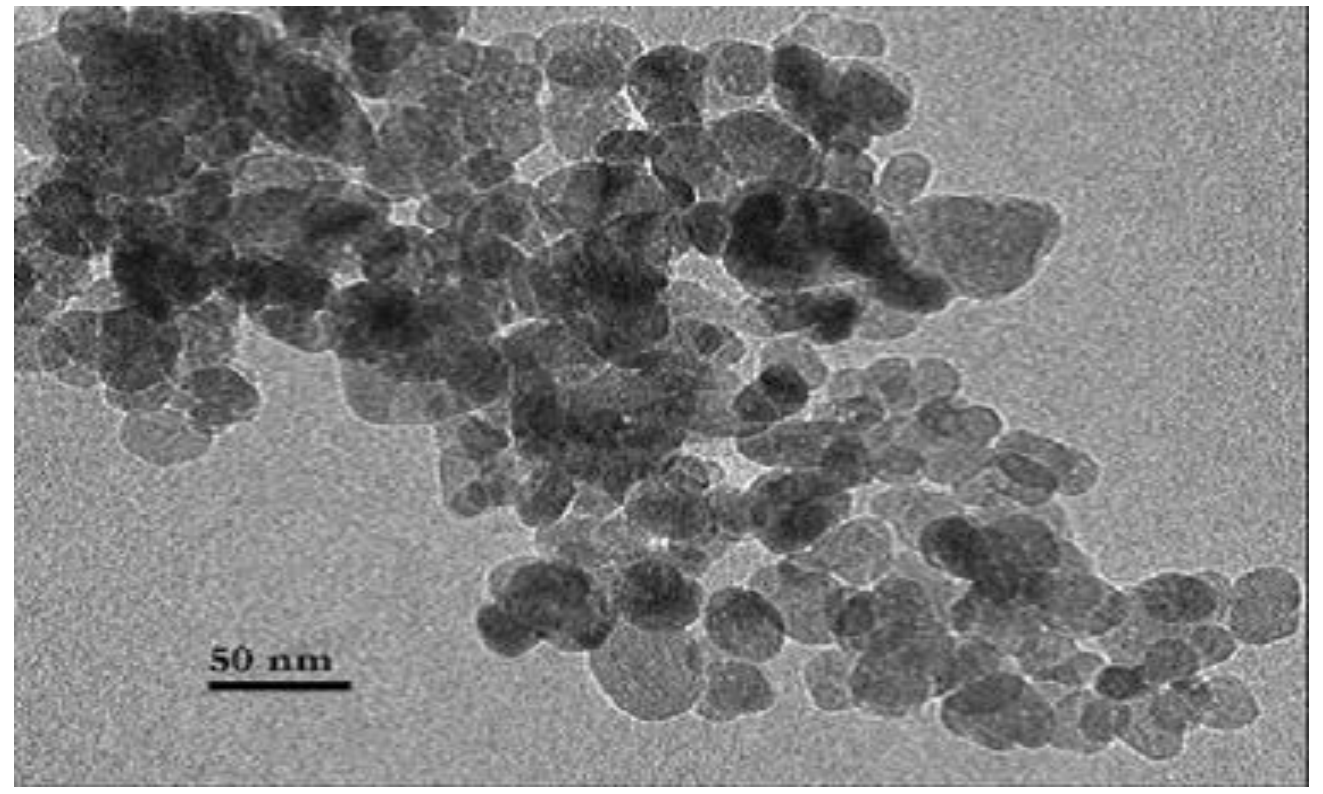

Figure 4 TEM Micrographs CdSe Quantum dots 


\section{CONCLUSIONS}

In conclusion, I have shown a new, simple method for the synthesis of high quality CdSe Quantum dots at normal temperature. The quantity of Se powder that used in experiment and the growing temperature in the reaction system plays an important role for the size control of the final product. On the basis of mechanism discussed above, this method should also be applicable to other selenides (i.e. I-V, IV-VI semiconductors). So that it is expected to be applicable to fabricate other nanosized semiconductor compounds. This route may open the way to the creation of other nano structures and avoid the use of high temperature.

\section{ACKNOWLEDGEMENT}

I acknowledge the condensed matter physics laboratory of department of physics, centre of advance studies, Banaras Hindu University for providing laboratory, financial support and thanks to Dr. Kedar Singh for his excellent support.

\section{REFERENCES}

1. L Brus, Quantum Crystallites and Nonlinear Optics. Appl. Phys, A53 (1991) 465.

2. Y Wang \& N Herron, Nanometer-Sized Semiconductor Clusters: Materials Synthesis, Quantum Size Effects and Photophysical Properties, J. Phys. Chem. 95 (1991) 525.

3. R Sathyamoorthy, V. Manjuladevi, P Sudhagar, S Senthilarasu \& U Pal, Surfactantassisted room-temperature synthesis of CdSe nanoclusters, Mater. Chem. Phys. 105 (2007) 20.

4. Wuister S F, Houselt A, Donega C M, Vanmaekelbergh, D \& Meijerink A, Temperature Antiquenching of the Luminescence from Capped CdSe Quantum Dots, Angew. Chem., Int. Ed, 43 (2004) 3029.

5. M Bruchez, M Moronne, $\mathrm{P}$ Gin, S Weiss \& A Poul Alivisatos, Semiconductor Nanocrystals as Fluorescent Biological Labels, Science, vol 281, no.5385, (1998) pp2013.

6. Catarina A, Esteves C \& Trindade T, Synthetic studies on II/VI semiconductor quantum dots, Current Opinion in Solid State \& Materials Science, 6 (2002) 347.

7. $\mathrm{T}$ Trindade, $\mathrm{P} \mathrm{O}^{\prime}$ Brien \& $\mathrm{N}$ Pickett, Nanocrystalline semiconductors: synthesis, properties and perspectives, Chem. Mater,13 (2001) 3843

8. D. xu, X Shi, GGuo, L Gui \& Y Tang, Electrochemical Preparation of CdSe Nanowire Arrays, J. Phys. Chim. B104 (2000) 5061.

9. C N R Rao, A Govindaraj, F Leonard Deepak, N A Gunari \& M Nath, Surfactant-assisted synthesis of semiconductor nanotubes and nanowires. Appl. Phys. Lett. 78 (2001) 1853. 
10. M Chen \& LGao, Synthesis and Characterization of Cadmium Selenide Nanorods Via Surfactant-Assisted Hydrothermal Method, J. Am. Ceram. Soc. 88 (2005) 1643.

11. Patterson, A L, The Scherrer Formula for X-Ray Particle Size Determination, Phys. Rev., 56 (1939) 978.

12. J. R. I. Lee, R. W. Meulenberg, K. M. Hanif, H. Mattoussi, J. E. Klepeis, L. J. Terminello, T. Buuren, Experimental Observation of Quantum Confinement in the Conduction Band of CdSe Quantum Dots, Phys. Rev., 98 (2007) 146803.

13. S. M. Kim, K. Kyhm, H. S. Yang, Optical Properties and Surface Conditions of CdSe Quantum Dots, J. Korean Phys. Soc. 49 (2006) 688. 